\title{
Ensuring a Safe and Qualitative Diagnostic Biopsy for Retroperitoneal Sarcomas
}

\author{
Kristin Kennedy Ambacher, BSc ${ }^{1}$; Andrea Marie Ibrahim, MSc ${ }^{2}$; Carolyn Nessim, MD, MSc, \\ FRCSC, FACS ${ }^{2,3}$
}

${ }^{1}$ Faculty of Medicine, University of Ottawa

${ }^{2}$ Ottawa Hospital Research Institute, The Ottawa Hospital

${ }^{3}$ Division of General Surgery, The Ottawa Hospital

A B STRACT

Retroperitoneal sarcomas represent one third of neoplasms in the retroperitoneum and as such are an important entity when evaluating masses in this area. They are often identified incidentally as they present with non-specific symptoms and are only detectable on physical exam when they have grown to a large size. This group of tumours is a challenge for physicians as it encompasses over 50 different histological subtypes and the course of treatment greatly depends on histopathological diagnosis. Biopsy of these lesions has recently become standard of care when evaluating a suspected retroperitoneal sarcoma. However, historically, there has been speculation over whether this practice promotes needle tract seeding resulting in local recurrence which has resulted in limited research on the topic. As such, there is a lack of literature describing the best parameters for a safe and effective biopsy of these lesions. Our ongoing research aims to identify biopsy parameters which yield a safe and qualitative diagnostic biopsy while minimizing complications and local recurrence with the goal of consistent and quality care for all patients presenting with retroperitoneal lesions.

\section{RÉ S U M É}

Les sarcomes rétropéritonéaux constituent un tiers des néoplasmes du rétropéritoine, et représentent ainsi une entité importante lors de l'évaluation de masses dans cet espace. Ils sont souvent découverts fortuitement puisqu'ils se manifestent par des symptômes non spécifiques et sont seulement décelables à l'examen physique lorsqu'ils atteignent une taille considérable. Ce groupe de tumeurs représente un défi pour les médecins, car il comprend plus de 50 différents sous-types histologiques et le traitement dépend largement du diagnostic histopathologique. La biopsie de ces lésions est récemment devenue la norme en matière de soins pour l'évaluation d'un sarcome rétropéritonéal soupçonné. Toutefois, par le passé, certains ont suggéré que cette pratique puisse possiblement disséminer le cancer et causer une récidive locale, ce qui a limité la recherche sur le sujet. Ainsi, il existe un manque de littérature décrivant les paramètres optimaux pour effectuer une biopsie sécuritaire et efficace de ces lésions. Notre recherche en cours vise à identifier les paramètres de biopsie qui produisent une biopsie diagnostique sécuritaire et qualitative, tout en minimisant les complications et les risques de récidive locale, dans le but de fournir des soins uniformes et de haute qualité à tous les patients avec des lésions rétropéritonéales.

\section{INTRODUCTION}

Soft-tissue sarcomas are a relatively rare group of malignancies of mesenchymal origin arising from soft-tissues such as fat, muscle, nerves and blood vessels. This is in contrast to a more common subtype of sarcoma arising from bone. Soft-tissue sarcomas arise most commonly in the extremities (i.e. upper and lower limbs) and retroperitoneum, the space between the peritoneum and posterior abdominal wall containing the kidneys, pancreas and other associated organs [1,2]. While soft-tissue sarcomas only make up $1 \%$ of all adult cancers, retroperitoneal sarcomas represent $15 \%$ of soft-tissue sarcomas and one third of retroperitoneal tumours in general, stressing their low incidence and the role of speciality care for masses in this region [2-4].

Keywords: Retroperitoneal; Sarcoma; Qualitative biopsy; Biopsy Parameters
Retroperitoneal sarcomas are often asymptomatic and identified incidentally during imaging performed for other purposes. This is mostly attributed to the large area encompassed by the retroperitoneal space which allows significant growth before a mass is noticeable or symptomatic. In fact, tumours in this space under 5 $\mathrm{cm}$ in size are rarely detected [5-7]. Furthermore, the most common presenting symptoms for patients with a retroperitoneal mass are non-specific (pain, weight loss, early satiety, nausea and a palpable abdominal mass) and often occur later in the course of the disease $[5,7]$. Luckily, despite its late detection, sarcomas are often identified without distant metastases because of their propensity for local extension, often pushing structures aside but rarely violating fascial planes [8]. In addition, soft-tissue sarcoma are known to have a disinclination for lymphatic spread which limits their metastatic potential $[9,10]$. It is due to this that the 
mainstay of treatment for retroperitoneal sarcoma is surgery with neo-adjuvant chemotherapy or radiation in certain cases $[11,12]$.

Retroperitoneal sarcomas present a challenge in terms of diagnosis and treatment plan due to the large diversity of histological subtypes that all present in similar fashion [2]. In fact, the World Health Organization recognizes over 50 subtypes of soft-tissue sarcoma. A further challenge with respect to these lesions is the large number of tumours that mimic sarcoma on presentation and imaging but which require very different treatment strategies. The most common example of this is lymphoma, which is often difficult to distinguish from sarcoma on CT scans and is treated by chemotherapy alone, whereas sarcoma is treated surgically [13].

Owing to the vast differential diagnosis of a retroperitoneal mass, it has become apparent that obtaining a histopathological diagnosis is a critical step in the work-up and management of these tumours. This paper describes the current practices in the evaluation of suspected retroperitoneal sarcoma, the role of needle biopsy and the historical controversy surrounding its use. We will also describe our current ongoing research at The Ottawa Hospital led by Dr. Carolyn Nessim, surgical oncologist, to standardize biopsy methods to ensure prompt diagnosis and proper treatment planning for patients with suspected retroperitoneal sarcoma.

\section{CURRENT MANAGEMENT PRACTICES}

Initial investigations of retroperitoneal tumours, as with most undiagnosed neoplasms, begin with radiologic imaging, usually CT scans or MRI. Imaging is useful for evaluating the size and extent of the mass as well as screening for lymphadenopathy or distant metastases. Moreover, imaging is required for surgical and/or radiation therapy planning $[11,14]$.

Following imaging, the common practice, and now standard of care, is to proceed with percutaneous biopsy of the mass $[11,15]$. This diagnostic approach is particularly important in suspected retroperitoneal sarcoma as although imaging can sometimes distinguish between a benign or malignant neoplasm, a histological diagnosis is important in dictating treatment plan [13]. Furthermore, treatment regimen and sequence can differ depending on the grade of the neoplasm. Core needle biopsy can differentiate between benign and malignant neoplasm with very high accuracy and correctly identifies the sarcoma histological subtype $88-90 \%$ of the time [3]. This allows the treating surgeon to distinguish between benign neoplasms that require observation and malignant sarcomas which require excision, as well as whether neo-adjuvant treatment is necessary for improving outcome.
For example, one of the most common types of retroperitoneal sarcoma is liposarcoma, a malignant soft-tissue sarcoma arising from adipose tissue that can present with well-differentiated or dedifferentiated cells. These two histological features are characterized by very different biologic behaviours and consequently require a different management plan [16]. Studies have only shown a reduction in local recurrence with neo-adjuvant radiation or chemotherapy for patients with dedifferentiated liposarcoma and not for patients with the well-differentiated subtype $[16,17]$.

Therefore, due to its key role in determining management strategy, core needle biopsy in the evaluation of retroperitoneal masses is now internationally practiced. Moreover, core needle biopsy is recommended by the Trans-Atlantic Retroperitoneal Sarcoma Working Group (TARPSWG), a working group established by multi-sarcoma-excellence institutions across the world in 2013 in order to develop consensus documents on the approach to treat this difficult disease [18]. The consensus guidelines strongly suggest image-guided percutaneous core needle biopsy to accurately sample the lesion. In addition, they recommend ensuring sampling of the more solid, dedifferentiated tumour components, as determined by well-perfused areas in contrast-enhanced CT scans or MRI.

\section{NEEDLE TRACT SEEDING}

Biopsy of retroperitoneal masses has been used with caution due to the belief that the procedure could lead to seeding of cancer cells along the needle tract, and thereby facilitate local recurrence. This has been shown in case reports pertaining to other types of cancers; however, relatively little data has been available with regards to risk of recurrence along the needle tract for retroperitoneal sarcoma [15]. Within the last five years, retrospective studies have demonstrated minimal risk of seeding, indicating this is not a reason to avoid biopsy [19-21]. In fact, a recent retrospective review from three tertiary sarcoma-treating centres (including our group at the Ottawa Hospital) looking at biopsy complications in 540 patients with a median follow-up of 50 months found only 2 patients $(0.37 \%)$ with a sarcoma recurrence in the presumed biopsy tract [22]. A separate study from Wilkinson et al. failed to identify a single patient in their 150-patient cohort who developed a biopsy-site recurrence. Furthermore, they did not identify any differences in local recurrence rates or overall survival when comparing patients who underwent biopsy to those who did not [21].

Biopsy of retroperitoneal sarcomas is also considered a safe procedure with minimal complications $[18,23]$. In the same 540 patient cohort mentioned previously, a second analysis described early complications of percutaneous biopsies of retroperitoneal masses in a subset of patients $(n=288)$. Specifically, $7(2.4 \%)$ bi- 


\section{Review \& Clinical Practice}

opsies resulted in minor bleeding with no transfusions required, $3(1 \%)$ patients reported significant pain which was managed effectively with acetaminophen and/or NSAIDs, and one patient $(0.3 \%)$ required an unplanned admission to the hospital for 24 hours [22].

Despite the fact that biopsy is now gaining support for being a safe method of pre-operative diagnosis of retroperitoneal masses, there is still a lack of literature surrounding this topic. Specifically, there is a knowledge gap regarding the quality measures which ensure not only a safe biopsy but one with high diagnostic yield (the likelihood the test will provide a diagnosis). To date, only one paper has made recommendations for biopsy of softtissue and bone sarcomas; however, of note, these recommendations did not focus specifically on retroperitoneal lesions [24]. In this study, the diagnostic yield (total number of biopsies that yield a diagnosis divided by total number of biopsies) was $77 \%$ for all lesions and there were no differences in yield according to needle gauge or imaging modality. This yield is relatively low when compared to studies in other tissues such as breast (100\%) and musculoskeletal tumors (91\%) $[25,26]$. Nonetheless, it is comparable to previous studies of retroperitoneal sarcoma ( $72 \%$ and $82 \%$ yield) and a study on chest wall sarcoma biopsies $(70 \%$ yield) $[23,27,28]$. The gap in the literature pertaining to biopsy of retroperitoneal sarcoma has therefore led our group to initiate a quality improvement audit of our tertiary centre.

\section{OUR RESEARCH}

As discussed, there are clear benefits to achieving histological diagnosis through biopsy of suspected retroperitoneal sarcomas when determining treatment plan, and these benefits favour patient safety and quality care improvement. That being said, no studies to date have examined the optimal method and parameters for sampling a suspected soft-tissue sarcoma originating in the retroperitoneum. Consequently, there is little literature to guide the clinician on the best practice for maximizing yield while minimizing risk of patient complications.

Our current research seeks to address this gap to provide guidelines for the safe and effective biopsy of retroperitoneal lesions. As retroperitoneal sarcomas are rare, we have collaborated with Mount Sinai Hospital in Toronto, a Cancer Care Ontario-designated Sarcoma centre, to obtain a large/representative sample size to retrospectively examine biopsy parameters of patients referred to our centres for suspected retroperitoneal sarcoma. The patient population in our study includes almost 400 patients who have undergone biopsy for suspected sarcoma at Mount Sinai Hospital or The Ottawa Hospital between 1999 and 2015. Patients were included in the retrospective review if they presented with a retroperitoneal mass, were older than 18 years of age and had a biopsy of the lesion. Patients were excluded if their lesion was suspected to be intra-peritoneal in origin or was not suspected to be a sarcoma. Pathology reports, diagnostic imaging study reports, and clinical consult and progress notes were used to record biopsy information for each patient in the study. These included lesion location, lesion composition based on diagnostic imaging, size of tumor, image-guidance modality for biopsy, biopsy needle gauge, number of specimens sent for pathological analysis, longest length of biopsy specimens, location where biopsy was performed (i.e. tertiary or primary care center), diagnosis at biopsy, whether the lesion is a recurrence or primary tumor, complications secondary to biopsy procedure, and final diagnosis based on post-operative pathology. Standard demographics were also collected. As mentioned above, we have previously investigated needle tract seeding in our patient cohort, therefore this data was not included in our current study.

Our next step is comparing diagnostic yield based on biopsy type (fine needle versus core), gauge size, number of passes, specimen size, and image-guidance modality. Most significantly, we are comparing the pathological diagnosis from the biopsy with the pathological diagnosis obtained from the final surgical specimen in order to calculate diagnostic accuracy. Our patient sample size is one of the largest of studies concerning retroperitoneal lesions; hopefully, this will provide support for our recommendations. This study is currently at the data analysis stage and the results will be reported in a future manuscript. The main goal of our study is to guide institutions towards standardization of retroperitoneal lesion biopsy protocols where quality and patient safety are prioritized.

\section{CONCLUSION}

Due to their rarity, little evidence-based data is available for surgical oncologists treating retroperitoneal sarcomas to base proper management of these tumours. Furthermore, due to past controversy over the safety of biopsy of these masses, there are no agreed upon and validated guidelines to direct sampling of these lesions. Specialized centres across the world have come together to form the Trans-Atlantic Retroperitoneal Sarcoma Working Group (TARPSWG) in order to gather maximum patient data of a rare disease, create guidelines on the diagnosis and surgical management of these tumours, and disseminate knowledge to physicians treating this disease. As a tertiary centre for sarcoma treatment, and as Dr. Carolyn Nessim is an active member of the TARPSWG, our overall goal as a centre is to contribute to the output of evidence-based data that can be trusted by clinicians to guide biopsy procedures in their practice. This will ultimately lead to consistent, quality, and safe care for all patients presenting with these lesions. 


\section{Review \& Clinical Practice}

\section{ACKNOWLEDGEMENTS}

We would like to thank Dr. Carol J Swallow, Sally Burtenshaw and Dr. David Berger-Richardson from Mount Sinai Hospital in Toronto for their collaboration on this study. We would also like to acknowledge the Lead of the Trans-Atlantic Retroperitoneal Sarcoma Working Group, Dr. Alessandro Gronchi, for his great leadership.

\section{REFERENCES}

1. Al-Absi E, Farrokhyar F, Sharma R, et al. A systematic review and meta-analysis of oncologic outcomes of pre- versus postoperative radiation in localized resectable soft-tissue sarcoma. Ann Surg Oncol. 2010;17(5):1367-74.

2. Van Roggen JFG, Hogendoorn PCW. Soft tissue tumours of the retroperitoneum. Sarcoma. 2000;4:17-26.

3. Strauss DC, Hayes AJ, Thomas JM. Retroperitoneal tumours: Review of management. Ann R Coll Surg Engl. 2011;93(4):275-80.

4. Canadian Cancer Society. Canadian Cancer Statistics 2016 [Internet]. Updated Oct. 2016 [cited 2017 Mar 7]. Available from: http://www.cancer.ca/ / media/cancer.ca/CW/cancer information/cancer 101/Canadian cancer statistics/Canadian-Cancer-Statistics-2016-EN.pdf?la=en

5. Solla JA, Reed K. Primary retroperitoneal sarcomas. Am J Surg. 1986;152(5):496-8.

6. Lewis JJ, Leung D, Woodruff JM, Brennan MF. Retroperitoneal soft-tissue sarcoma: analysis of 500 patients treated and followed at a single institution. Ann Surg. 1998;228(3):355-65.

7. Windham TC, Pisters PWT. Retroperitoneal sarcomas. Cancer Control. 2005;12(1):36-43.

8. Potter DA, Glenn J, Kinsella T, et al. Patterns of recurrence in patients with high-grade soft-tissue sarcomas. J Clin Oncol. 1985;3(3):353-66.

9. Mazeron JJ, Suit HD. Lymph nodes as sites of metastases from sarcomas of soft tissue. Cancer. 1987;60(8):1800-8.

10. Blazer III DG, Sabel MS, Sondak VK. Is there a role for sentinel lymph node biopsy in the management of sarcoma? Surg Oncol. 2003;12(3):201-6.

11. European Sarcoma Network Working Group. Soft tissue and visceral sarcomas: ESMO clinical practice guidelines for diagnosis, treatment and followup. Ann Oncol. 2014;25(Supplement 3):iii102-iii112.

12. Karakousis CP, Gerstenbluth R, Kontzoglou K, Driscoll DL. Retroperitoneal Sarcomas and Their Management. Arch Surg. 1995;130:1104-9.

13. Francis IR, Cohan RH, Varma DG, Sondak VK. Retroperitoneal sarcomas. Cancer Imaging. 2005;5:89-94.

14. Arca MJ, Sondak VK, Chang AE. Diagnostic procedures and pretreatment evaluation of soft tissue sarcomas. Semin Surg Oncol. 1994;10(5):323-31.

15. Berger-Richardson D, Swallow CJ. Needle tract seeding after percutaneous biopsy of sarcoma: Risk/benefit considerations. Cancer. 2016.

16. Lahat $\mathrm{G}$, Madewell JE, Anaya DA, et al. Computed tomography scan-driven selection of treatment for retroperitoneal liposarcoma histologic subtypes. Cancer. 2009;115(5):1081-90.

17. Pham V, Henderson-Jackson E, Doepker MP, et al. Practical Issues for Retroperitoneal Sarcoma. Cancer Control 2016;23(3):249-64.

18. Trans-Atlantic RPS Working Group. Management of Primary Retroperitoneal Sarcoma (RPS) in the Adult: A Consensus Approach From the Trans-Atlantic RPS Working Group. Ann Surg Oncol. 2015;22(1):256-63.

19. Miah AB, Hannay J, Benson C, et al. Optimal management of primary retroperitoneal sarcoma: an update. Expert Rev Anticancer Ther. 2014;14(5):56579.

20. Strauss DC, Hayes AJ, Thomas JM. Preoperative core needle biopsy is safe and does not affect oncological outcome in retroperitoneal sarcoma. CTOS 17th Annu Meet. 2012.

21. Wilkinson MJ, Martin JL, Khan AA, Hayes AJ, Thomas JM, Strauss DC. Percutaneous core needle biopsy in retroperitoneal sarcomas does not influence local recurrence or overall survival. Ann Surg Oncol. 2015;22(3):853-8.

22. Berger-Richardson D, Nessim C, Burtenshaw S, Gladdy R, Gronchi, A, Fiore M SC. Early and Late Complications of Percutaneous Biopsy of Retroperitoneal Masses at Three Tertiary Sarcoma Centers. In: Society of Surgical Oncology. 2017.
23. Hwang SY, Warrier S, Thompson S, Davidson T, Yang JL, Crowe P. Safety and accuracy of core biopsy in retroperitoneal sarcomas. Asia Pac J Clin Oncol. 2016;12(1):e174-8.

24. Wu JS, Goldsmith JD, Horwich PJ, Shetty SK, Hochman MG. Bone and softtissue lesions: what factors affect diagnostic yield of image-guided coreneedle biopsy? Radiology. 2008;248(3):962-70.

25. Fishman JE, Milikowski C, Ramsinghani R, Velasquez MV, Aviram G. US-guided core-needle biopsy of the breast: How many specimens are necessary? Radiology. 2003;226(3):779-82.

26. Carrino J, Khurana B, Ready J, Silverman S, Winalski C. Magnetic Resonance Imaging-Guided Percutaneous Biopsy of Musculoskeletal Lesions. J Bone Jt Surg Am. 2007;89(10):2179-87.

27. Kachroo P, Pak PS, Sandha HS, et al. Chest Wall Sarcomas are Accurately Diagnosed by Image-Guided Core Needle Biopsy. J Thorac Oncol. 2012;7(1):151-6.

28. van Dalen $T$, van Coevorden F, van Geel A, Hoekstra H, Hennipman A. A biopsy of a suspected soft tissue sarcoma in the retroperitoneal space; the diagnostic yield and the risk of contamination of the different procedures. Eur J Cancer. 2001;37:S84. 